\title{
Stimuli and incentives as determinants of the successive negative contrast effect*
}

\author{
JAMES H. McHOSE \\ Southern Illinois University, Carbondale, Ill. 62901
}

Seventy-two rats received 10 preshift and 7 postshift instrumental conditioning trials in a straight runway. The preshift magnitudes of intertrial reinforcement and runway reward were factorially manipulated, while all Ss received a small ITR and runway reward in the postshift period. Both reductions in ITR and runway reward magnitude produced negative contrast effects.

In simple instrumental conditioning, the performance of rats initially consistently reinforced with a large reward and subsequently shifted to a small reward is temporarily depressed relative to that of Ss trained and maintained on small reward (cf. Black, 1968). There are at least two different theoretical viewpoints concerning the experimental variable responsible for this depression or negative contrast effect (NCE), one based on considerations of the incentive properties of reinforcement, the other on the stimulus properties of reinforcement. Incentive theories, exemplary of which are frustration-based viewpoints (e.g., DiLollo \& Beez, 1966), attribute the NCE to a discrepancy between the amount of contingent reinforcement regularly received in the preshift period and the amount received in the postshift period. Stimulus frameworks assume that the NCE results not from a change in response-contingent reward per se, but from a change in the stimuli produced by these reinforcement events. Capaldi's (1967) stimulus-specificity framework, for example, assumes that different reward amounts occasion different stimuli which remain functional until the next conditioning trial and thus eventually become part of the stimulus complex to which the instrumental response is attached. Within this framework, the NCE occurs when Ss trained to respond to the stimulus associated with large reward, $\mathrm{SL}^{\mathrm{L}}$, in the preshift period are suddenly presented with a (different) stimulus associated with small reward, $S^{S}$.

For cases in which Ss receive consistent reward amounts from trial to trial in the preshift period and a different constant reward in postshift, the roles of stimulus and incentive properties of the reinforcer are obviously inseparable, since they are determined by the same experimental event, i.e., the reward delivered subsequent to the instrumental response. Recently, however, Calef (1972) attempted to separate these factors by the factorial manipulation of the amount of "reward" received prior to response (stimulus) and the amount of reward received immediately following

*Supported by Research Grant NSF 30510 from the National Science Foundation. response (incentive). Changes in the stimulus factor, manipulated through the intertrial reinforcement (ITR) procedures developed by Capaldi and his associates (Capaldi, Hart, \& Stanley, 1963; Capaldi \& Olivier, 1967), proved unrelated to the NCE; only incentive changes resulted in an NCE.

It would seem that the separation of stimulus and incentive factors in the context of consistent reinforcement schedules can only be accomplished by use of the Calef (1972) technique. Nevertheless, the extent to which Calef's data contradict current (Capaldi, 1967) stimulus interpretations of the NCE clearly hinges upon the extent to which an ITR delivered between two trials regulates the reward-magnitude-related stimulus available on the second trial. In this context, it can be argued that since Ss received 36 ITRs and rewards in the preshift period of the Calef study, they came to discriminate between ITRs and regular rewards, with the result that the magnitude-specific stimulus present on the second of two conditioning trials was determined by the regular reward event of the first trial, not by the immediately preceding ITR. Calef's (1972) findings would thus be consistent with a stimulus-specificity analysis.

While there are apparently no data directly relevant to the assertion that rats can discriminate ITRs from regular rewards, there are indeed data consonant with the set of theoretical assumptions comprising this hypothesis and the various other assumptions of sequential theory (e.g., Capaldi \& Olivier, 1967).

The present study employs the Calef (1972) technique for separating the stimulus and incentive contributions to the NCE, but with fewer preshift training trials. Reducing preshift exposure to ITRs and regular rewards presumably would preclude the formation of a discrimination between these two events (cf. Capaldi \& Olivier, 1967), with the result that the separated stimulus factor (ITR) would be clearly relevant to a stimulus-specificity analysis of the NCE.

\section{METHOD}

The Ss were 72 naive male albino rats, approximately 90 days old at the beginning of the experiment, obtained from the Holtzman Company, Madison, Wisconsin.

The runway apparatus consisted of a startbox (SB), alley, and goalbox (GB) section, all painted flat black. The SB, alley, and GB sections were 10,32 , and $10 \mathrm{in}$. long, respectively, with an interior width of $3.5 \mathrm{in}$. and interior height of $4 \mathrm{in}$. Opaque doors separated the SB and goal sections from the alley segment of the apparatus. Photocell clock circuitry provided traversal times over the first three consecutive 12 -in. segments of the alley-goal sections. A fan mounted on the exterior wall of the goalbox extracted air from the goal section via $1 / 8$-in. holes in the rear wall.

Fourteen days prior to the first experimental day, Day 15, all 


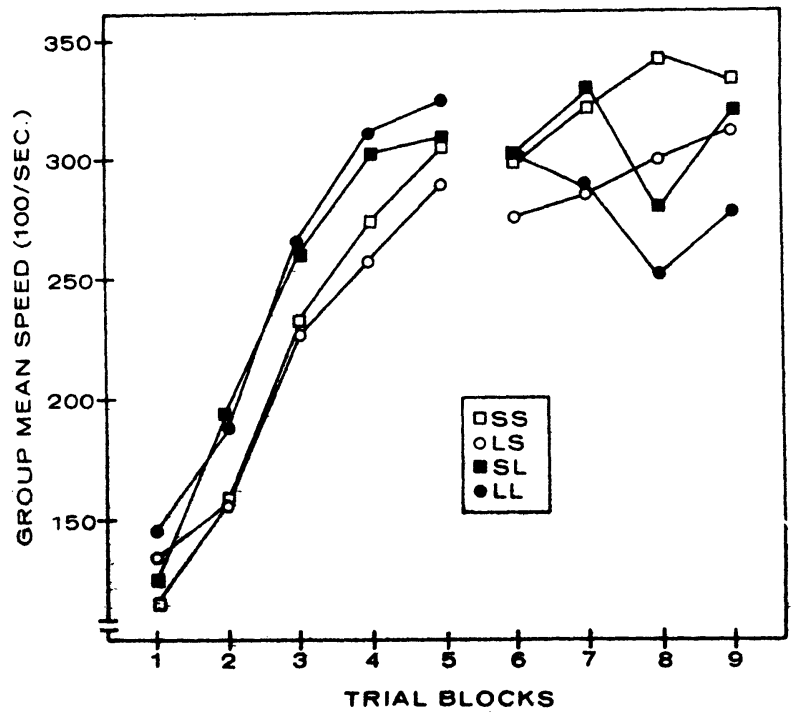

Fig. 1. Group mean goal speeds as a function of trial blocks.

Ss were placed on a 23-h food-deprivation regimen which was maintained throughout the study. On Days 7-14, Ss were handled approximately 2 min each. On Days 13-14, Ss were allowed to explore the start and run sections of the runway and were fed $1 \mathrm{~g}$ of $45-\mathrm{mg}$ Noyes pellets, identical to the subsequent reinforcement pellet, in addition to their daily food ration.

The experiment consisted of a 10-trial preshift period and a 7-trial postshift period, with each trial preceded by a stimulus placement or intertrial reinforcement (ITR). Trials were administered at the rate of one per day, with the stimulus placement for any one trial occurring as the last event of the preceding day. The design was a factorial manipulation of the amount of reward regularly received on each preshift runway trial (16 or 2 pellets) and the amount of ITR (16 or 2 pellets) preceding each trial. During the postshift period, all Ss always received two-pellet runway reward preceded by a two-pellet ITR. In each of two replications, nine Ss were assigned to each of four groups, labeled according to the magnitudes of ITR (large, L, or small, S) and runway rewards regularly received during the preshift period: Groups SS, SL, LS, and LL.

On each day, with the exception of the first training day, the runway trial was administered to each of $36 \mathrm{Ss}$ before the first S received its ITR. This procedure resulted in a trial-ITR interval of approximately $25 \mathrm{~min}$. On the first day of training, Ss received only an ITR.

An individual runway trial began with the placement of $S$ in the startbox. After $S$ had oriented to the start door for $3 \mathrm{sec}$, the door was opened and, following Ss entry of GB, the retrace door was closed and $\mathrm{S}$ removed from GB immediately following food consumption. On ITR placements, S was placed in GB, with the retrace door closed, and removed from GB immediately after food consumption.

\section{RESULTS}

As in the Calef (1972) study, the most pronounced depression or negative contrast effects occurred in the third response measure, the goal measure. Group mean goal speeds are plotted as a function of trial blocks in Fig. 1. Each trial block contains the data for two trials (days), with the exception of Blocks 11 and 12, each of which contains the data for one trial (day) only.

As can be seen in Fig. 1, performance during the middle portion of the preshift period was directly related to incentive magnitude, Groups $\mathrm{LL}$ and $\mathrm{SL}$ displaying faster speeds than Groups LS and SS. Variance analysis of the data for Blocks 3 and 4, containing incentive magnitude, stimulus magnitude, and replications as factors, yielded a significant incentive effect, $F(1,64)=7.16, p<.01$. Neither the stimulus nor the stimulus by incentive effects were statistically reliable. A similar analysis of the data for Block 5, however, yielded no significant effects of either stimulus or incentive factors, indicating that the effects of incentive magnitude had largely dissipated by Block 5 .

Turning to the effects of reductions in stimulus and incentive magnitudes on postshift performance, inspection of Fig. 1 indicates that magnitude reductions of either variety were sufficient to depress postshift performance. The effects of a reduction in stimulus magnitude alone are apparent beginning at Block 7, with the performance of Groups LS and LL depressed relative to that of Groups SS and SL, respectively. Analysis of variance of the data for Block 7 yielded a significant (preshift) stimulus effect, $F(1,64)=4.53, p<.05$. Similarly, the effects of a reduction in incentive magnitude alone are first apparent at Block 8, with the performance of Groups SL and LL depressed relative to that of Groups SS and LS, respectively. Analysis of variance of the data for Block 8 yielded a significant (preshift) incentive magnitude effect, $F(1,64)=12.43$, $\mathrm{p}<.01$, as well as a reliable effect of preshift stimulus magnitude, $F(1,64)=8.94, p<.01$.

While the replications factor in each of the preceding analyses was statistically reliable $(p<.01), F$ ratios for interactions of the replications factor with incentive and stimulus factors were uniformly less than unity.

\section{DISCUSSION}

The present experiment was designed to determine whether, with minimal training, reductions in intertrial reinforcement (ITR) magnitude or reductions in incentive magnitude would produce a negative contrast effect (NCE). The data indicate that a reduction in either ITR or incentive magnitude is sufficient to produce an NCE. Thus, Group LS, which experienced only a reduction in ITR, and Group SL, which experienced only an incentive reduction, displayed slower speeds in the postshift period than did Group SS. The observation that Group LL displayed the lowest postshift performance level indicates that reductions in ITR and incentive magnitudes combined to enhance the negative contrast effect.

With more exposures to ITR and runway reward than were used in this study, only incentive reductions produce the NCE (Calef, 1972). The present data in conjunction with Calef's findings thus suggest that either ITR or runway magnitude reductions are sufficient to produce the NCE after a minimal exposure to these reward events but that, following repeated exposure to these events, only a reduction in runway reward produces an NCE. The theoretical significance of this pattern of results depends upon the theoretical interpretation of the function of an ITR.

Assume with Capaldi \& Olivier (1967) that an ITR sets the reward-related stimulus characteristic available for conditioning on the following instrumental trial. A condition such as Group LS would acquire habit to $S^{L}$, the stimulus associated with large reward, during the preshift period. A reduction in ITR 
magnitude exposes $S$ to the stimulus associated with small reward, SS. Since only generalized habit is available to $S^{S}$, Group LS performance during the postshift period should be depressed relative to that of Group SS. Within stimulus-specificity theory, the NCE displayed by Group LS may be interpreted in the same fashion as the NCE attending shifts from large to small reward in the conventional successive contrast literature (cf. Capaldi, 1967). Within this framework, the observation that ITR reductions do not produce an NCE after more extended training (Calef, 1972) may be attributed to the development of a discrimination between ITR and runway reward events, with the consequence that ITRs no longer possess a stimulus replacement function (Capaldi \& Olivier, 1967).

While the observed effects of ITR reduction of the NCE in the present data, as well as in Calef's (1972) study, are readily compatible with stimulus-specificity theory, the present observation that incentive (runway reward) reductions alone were sufficient to produce the NCE is not. If Ss fail to discriminate between ITRs and runway rewards, only ITRs should function as (reinforcement magnitude) stimuli in the present experiment, with the consequence that the instrumental response for Group SL would be attached to $S^{S}$, the stimulus which is also present during the postshift period. When there is no stimulus change, there should, of course, be no NCE from a sequential viewpoint.

Perhaps the best fashion in which to view the present results in conjunction with those reported by Calef (1972) is to assume with Capaldi \& Olivier (1967) that Ss do indeed come to discriminate between ITRs and runway rewards after repeated exposures to these events but that the NCE is an incentive-related phenomenon. Thus, reductions in incentive (runway reward) magnitude would be expected to produce NCEs in the Calef (1972) study and in the present study. Reductions in ITR magnitude would suffice to produce NCEs prior to the development of a discrimination (the present data), because they function to increase incentive or expectancy $\left(r_{g}\right)$ levels, but not after the formation of a discrimination.

Finally, it should be noted that in the Calef (1972) study only groups which received a large magnitude ITR in the preshift period developed NCEs following a reduction in incentive magnitude, while both Groups LL and SL displayed NCEs in the present study. Calef interpreted his findings as indicating that $\mathrm{Ss}$ with small ITRs (and large incentives) were frustrated on ITR trials and that this frustration was conditioned to the locomotion response, thus precluding any frustration-engendered interference (and NCE) following a reduction in incentive. If one chooses to operate within this theoretical framework, the fact that the present Group SL displayed an NCE may be said to reflect the fact that frustration-produced stimuli were not conditioned to the locomotor response in the present study due to the minimal preshift training (cf. Amsel, 1967).

\section{REFERENCES}

Amsel, A. Partial reinforcement effects on vigor and persistence. In K. W. Spence and J. T. Spence (Eds.), The psychology of learning and motivation. Vol. 1. New York: A cademic Press, 1967.

Black, R. W. Shifts in magnitude of reward and contrast effects in instrumental and selective learning: A reinterpretation. Psychological Review, 1968, 75, 114-126.

Calef, R. S. The effect of large and small magnitudes of intertrial reinforcement on successive contrast effects. Psychonomic Science, 1972, 29, 309-312.

Capaldi, E. J. A sequential hypothesis of instrumental learning. In $\mathrm{K}$. W. Spence and J. T. Spence (Eds.), The psychology of learning and motivation. Vol. 1. New York: Academic Press, 1967.

Capaldi, E. J., Hart, D., \& Stanley, L. R. Effect of intertrial reinforcement on the aftereffect of nonreinforcement and resistance to extinction. Journal of Experimental Psychology, $1963,65,70-74$.

Capaldi, E. J., \& Olivier, W. P. Effect of intertrial reinforcement following a substantial number of consistently rewarded trials. Journal of Experimental Psychology, 1967, 75, 135-138.

DiL ollo v. \& Beez, V. Negative contrast effect as a function of magnitude of reward decrement. Psychonomic Science, 1966. 5, 99-100.

(Received for publication January 15, 1973.) 